\title{
An Improved Target Location Algorithm for General Airborne Bistatic SAR System
}

\author{
Xin Zhou, Yong Li, Ya Li \\ College of Electronic and Information Engineering \\ Nanjing Univ. of Aeronautics and Astronautics, Nanjing, China \\ limack@nuaa.edu.cn
}

\begin{abstract}
In this paper, we present a modified imaging algorithm and the target-location method for the general case of bistatic synthetic aperture radar (BiSAR), when the transmitter and receiver move along nonparallel trajectories with different velocities. As a step to a numerically efficient processor, the basic linear range-Doppler (LRD) algorithm is firstly generalized from the monostatic SAR to BiSAR imaging, which also enable the ground-target location processing to be feasible. We show that, the LRD algorithm combined with the Newton iteration method could offer a precision and efficient approach by solving the double hyperbolic equation of the range Doppler history of the general BiSAR system. Simulation results of airborne BiSAR imaging and location of stationary ground point-targets are given to demonstrate the effectiveness of the proposed algorithm.
\end{abstract}

Index Terms-Bistatic SAR, Liner Range-Doppler, The Newton iteration method.

\section{INTRODUCTION}

Bistatic synthetic aperture radar (BiSAR) systems, as opposed to monostatic SAR, offer some degrees of freedom in choosing transmitter (illuminator) and (passive) receiver motion trajectories [1]. The bistatic configuration, when transmitter and receiver move along nonparallel tracks with unequal velocities, is called the general BiSAR. This kind of BiSAR system has the potential for various applications [2][3]. One of them is the ground-target location in BiSAR images, which is necessary for ensuring the geometry calibration accuracy and effective understanding and interpretation of the radar images.

The linear range Doppler (LRD) algorithm is a computational efficient algorithm, which was originated from the basic theory of rotating-object imaging [4]. The LRD algorithm has been tested to be robust for mid-resolution monostatic SAR imaging, because the whole processing is achieved by fast Fourier transform and vector-multiplication operations. The original LRD algorithm needs to be modified before being used in processing BiSAR data, because there are distinct differences in the phase history of the received signal and the sample location of spatial frequency.

As discussed in this paper, the range-Doppler history of the general bistatic configuration is a double hyperbolic equation. To solve such a nonlinear equation, we choose to use the Newton iteration method because of its robustness and higher rate of convergence, particularly in handling the strongly nonlinear problems [5][6].

This paper is organized as follows: In section II, the geometric model of the bistatic SAR, the range history and the Doppler shift are analyzed. In section III, the mathematic expressions are derived using the Newton iteration method. Section IV shows the simulation results followed by a conclusion.

\section{RANGE HISTORY FORMULATION OF BISAR}

We consider a spotlight BiSAR system operating in a general geometry shown in Fig.1. For simplicity, the transmitter and receiver are assumed to travel along two nonparallel straight lines with constant forward velocities of $V_{t}$ and $V_{r}$ respectively. The central ground point of the scene $\mathrm{O}$ is defined as the origin of the coordinate system. Related to point $\mathrm{O}$, the initial spatial squint angles respectively are $\theta_{t}$ and $\theta_{r}$ at the center of the aperture.

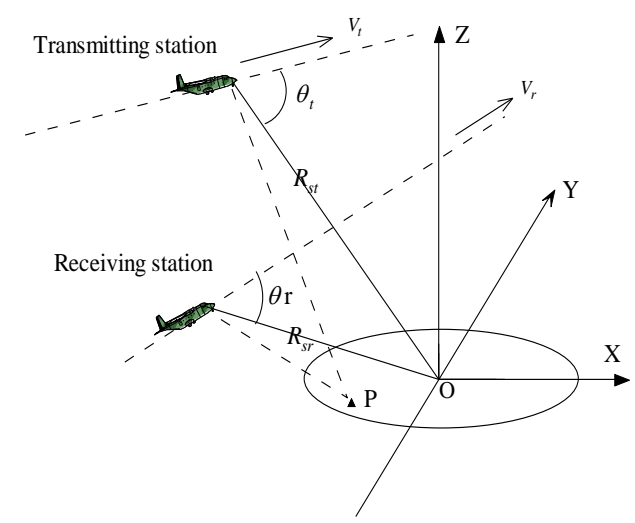

Fig. 1. Imaging geometry of an airborne general BiSAR system

Let $t$ represent the slow time in azimuth direction, then the instantaneous slant range between radar antenna phase center (APC) and scene center $\mathrm{O}$ is

$$
R_{o}(t)=R_{o r}(t)+R_{o t}(t) \quad-\frac{T_{a}}{2} \leq t \leq \frac{T_{a}}{2}
$$


where

$$
\left\{\begin{array}{l}
R_{o t}(t)=\sqrt{R_{s t}^{2}-2 V_{t} \cdot t \cdot R_{s t} \cdot \cos \left(\theta_{t}\right)+\left(V_{t} \cdot t\right)^{2}} \\
R_{o r}(t)=\sqrt{R_{s r}{ }^{2}-2 V_{r} \cdot t \cdot R_{s r} \cdot \cos \left(\theta_{r}\right)+\left(V_{r} \cdot t\right)^{2}}
\end{array}\right.
$$

and Ta is the synthetic aperture time, $R_{s t}=R_{o t}(0), R_{s r}=R_{o r}(0)$ represent the initial ranges from the APCs of the transmitter and receiver to the central point $\mathrm{O}$ at the aperture center respectively.

Assuming $\left(x_{p}, y_{p}, z_{p}\right)$ is the coordinate of an arbitrary scatterer $\mathrm{P}$ in the scenario, the instantaneous slant range from the APC to the scatterer $\mathrm{P}$ is

$$
\begin{aligned}
& R(t)=\sqrt{\left(X_{t}-x_{p}\right)^{2}+\left(Y_{t}-y_{p}\right)^{2}+\left(Z_{t}-z_{p}\right)^{2}} \\
& +\sqrt{\left(X_{r}-x_{p}\right)^{2}+\left(Y_{r}-y_{p}\right)^{2}+\left(Z_{r}-z_{p}\right)^{2}}
\end{aligned}
$$

where $\left(X_{t}, Y_{t}, Z_{t}\right)$ and $\left(X_{r}, Y_{r}, Z_{r}\right)$ represent respectively the 3-D instantaneous positions of the transmitter and the receiver.

$$
r_{p}=\sqrt{x_{p}^{2}+y_{p}^{2}+z_{p}^{2}}
$$

$R(t)$ can be decomposed as the summation $R_{o}(t)$ and a residue component as

$$
R(t)=R_{o}(t)+\Delta_{r}(t)
$$

Considering $R_{o t}(t) \square r_{p}, R_{o r}(t) \square r_{p}$, by using the Taylor series expansion method and ignoring their high order terms, we can get

$$
\begin{aligned}
\Delta_{r}(t) & =-r_{p} \cdot \cos \alpha_{t}(\mathrm{t})-r_{p} \cdot \cos \alpha_{r}(\mathrm{t}) \\
& =-r_{p} \cdot \cos \left[\alpha_{t}(0)+\Omega_{t} \cdot t\right]-r_{p} \cdot \cos \left[\alpha_{r}(0)+\Omega_{r} \cdot t\right] \\
& =x_{p} \cdot \frac{\cos \left[\alpha_{t}(0)+\Omega_{t} \cdot t\right]}{\cos \varphi}-y_{p} \cdot \frac{\cos \left[\alpha_{r}(0)+\Omega_{r} \cdot t\right]}{\sin \varphi}
\end{aligned}
$$

and

$$
\Omega_{t} \approx \frac{V_{t} \cdot \sin \left(\alpha_{t}\right)}{R_{s t}}, \Omega_{r} \approx \frac{V_{r} \cdot \sin \left(\alpha_{r}\right)}{R_{s r}}
$$

where $\alpha_{t}$ and $\alpha_{r}$ represent respectively the angels between the radar-beam pointing vector and OP of the transmitter and the receiver. The residue part $\Delta_{r}(t)$ in equation (5) represents the rotational component of scatterer $\mathrm{P}$ relative to the scene central $\mathrm{O}$, which is useful to the imaging.

After the range compression, the echo signal can be expressed as

$$
\begin{aligned}
r(t, \tau)= & G(t) \sin c\left\{\pi B\left[\tau-\frac{\left(R_{o}(t)+\Delta_{r}(t)\right)}{c}\right]\right\} \\
& \cdot \exp \left\{-j \frac{2 \pi}{\lambda}\left[R_{o}(t)+\Delta_{r}(t)\right]\right\}
\end{aligned}
$$

where $\tau$ represents the fast time (range time), $\lambda$ is the wave length, $\mathrm{c}$ is the velocity of microwave propagation, $G(\mathrm{t})$ represents the weight of the antenna to the echo signal.

The next processing in LRD algorithm is the motion compensation which includes two steps, i.e., the range alignment and the phase compensation. The point is translating the motion between airborne and the imaging scene into the rotating motion. The purpose of the range alignment is to remove the range displacement caused by $R_{o}(t) / c$ in the $\tau$ direction in equation (8) pulse by pulse, while aim of phase compensation is to remove the phase term $\exp \left\{-j \frac{2 \pi R_{o}(t)}{\lambda}\right\}$ by multiplying its conjugate counterpart.

After motion compensation, The Doppler frequency centroid $f_{d c p}$ of slant range history of the scatterer $\mathrm{P}$ can be formulated as

$$
f_{d c p}=-\frac{1}{\lambda}\left[-x_{p} \cdot \Omega_{t} \cdot \frac{\mathrm{si} \operatorname{mo} x_{t}(0)}{\cos \varphi}+y_{p} \cdot \Omega_{r} \cdot \frac{\operatorname{si~} 1 \alpha_{r} \quad(\rho}{\sin \varphi}\right]
$$

Equation (6) and (9) show the basic relationships how the coordinate of the scatterer $\mathrm{P}$ is determined by the range delay and the Doppler frequency of the received signal.

\section{LOCATION METHOD OF BISAR}

One of the important tasks of the SAR is to discover the targets, accomplish the battlefield environment surveillance and prepare for destroying and setting down a plan, hence how to improve the quality of the targets and positioning the targets properly become significant [7]. What is introduced in this section is the Newton iteration method which is a method used to solve the nonlinear equations. Here, the Newton iteration method is used to pinpoint targets based on the BiSAR.

The functions of each step of imaging are explained as following:

1) Range compression, as the match filter, in order to obtain high range-resolution;

2) Motion compensation (relative to the reference point) mainly includes the Range alignment and phase compensation. The aim of this step is translate the motion between airborne and the imaging scene into the standard rotating motion;

3) Azimuth focusing processing, in order to obtain the focusing imaging;

4) Geometric distortion correction, in order to correct geometric distortion.

The equations used to describe the Doppler shift is

$$
\left\{\begin{array}{l}
\cos \left(\theta_{t}\right)=\frac{V_{t x} \cdot\left(x_{o}-X_{t}\right)+V_{t y} \cdot\left(y_{o}-Y_{t}\right)+V_{t z} \cdot\left(z_{o}-Z_{t}\right)}{\left|V_{t}\right| \cdot \sqrt{\left(x_{o}-X_{t}\right)^{2}+\left(y_{o}-Y_{t}\right)^{2}+\left(z_{o}-Z_{t}\right)^{2}}} \\
\cos \left(\theta_{r}\right)=\frac{V_{r x} \cdot\left(x_{o}-X_{r}\right)+V_{r y} \cdot\left(y_{o}-Y_{r}\right)+V_{r z} \cdot\left(z_{o}-Z_{r}\right)}{\left|V_{r}\right| \cdot \sqrt{\left(x_{o}-X_{r}\right)^{2}+\left(y_{o}-Y_{r}\right)^{2}+\left(z_{o}-Z_{r}\right)^{2}}} \\
f_{\text {dco }}=\frac{1}{\lambda} \cdot\left[V_{t} \cdot \cos \left(\theta_{t}\right)+V_{r} \cdot \cos \left(\theta_{r}\right)\right]
\end{array}\right.
$$

The diagram in Fig. 2 with two parts includes the imaging process based on LRD algorithm and target location process using Newton iteration method [4-12]. 


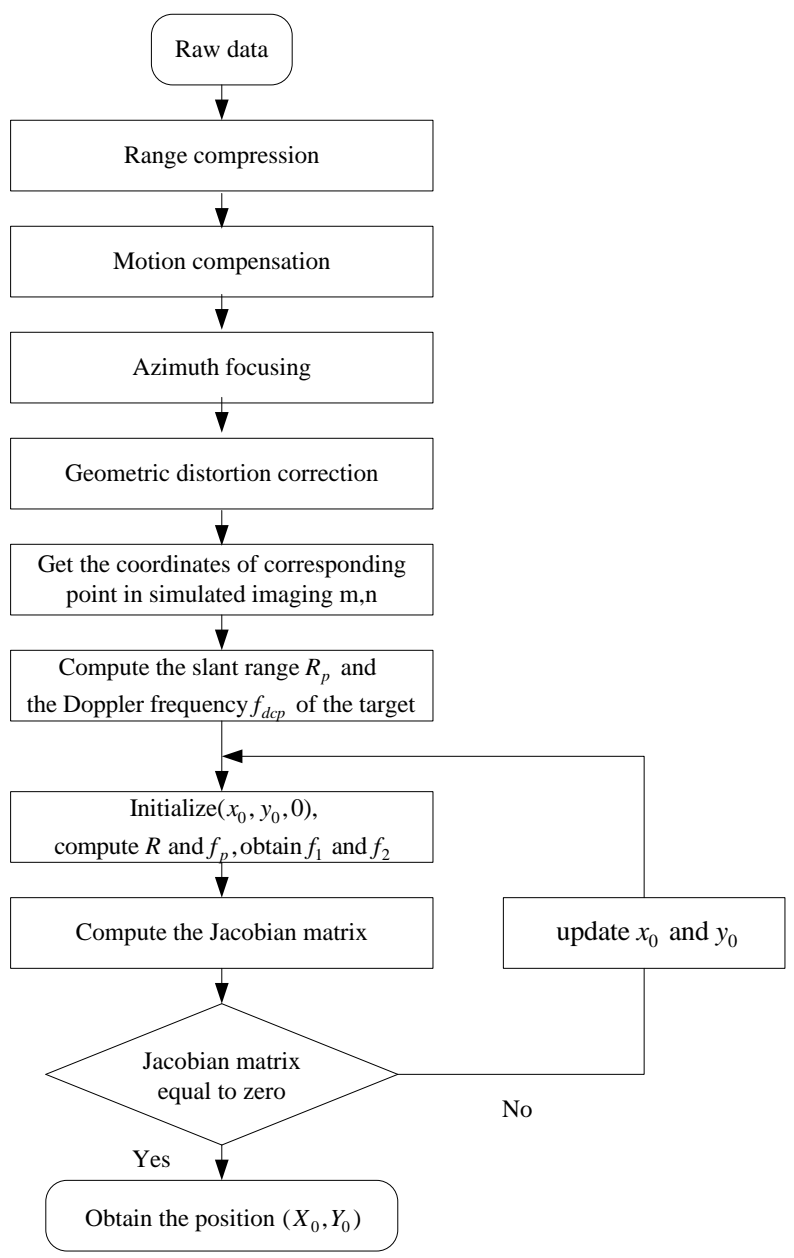

Fig. 2. Functional block diagram of the imaging and target location

The range between the radar APC to the target $R_{p}$ and the Doppler frequency centroid $f_{d c p}$ can be expressed in the BiSAR image as

$$
\left\{\begin{array}{l}
R_{p}=\left(n-\frac{N_{r}}{2}\right) \cdot p_{r}+R_{r e f} \\
f_{d c p}=\left(m-\frac{N_{a}}{2}\right) \cdot \frac{P R F}{N_{a}}+f_{d c o}
\end{array}\right.
$$

Non-linear equations can be solved using the Newton iteration method. First, we have to initialize $\left(x_{0}, y_{0}, z_{0}\right)$, and $f_{d}$ can be computed according to equation (10).

Let

$$
\left\{\begin{array}{l}
f_{1}(x, y)=R_{p}-R \\
f_{2}(x, y)=f_{d c p}-f_{d}
\end{array}\right.
$$

where

$$
\begin{aligned}
R= & \sqrt{\left(X_{t}-x_{0}\right)^{2}+\left(Y_{t}-y_{0}\right)^{2}+\left(Z_{t}-z_{0}\right)^{2}} \\
& +\sqrt{\left(X_{r}-x_{0}\right)^{2}+\left(Y_{r}-y_{0}\right)^{2}+\left(Z_{r}-z_{0}\right)^{2}}
\end{aligned}
$$

Here, let $z_{0}=0$, then compute the Jacobian matrix, if

$$
\operatorname{det}\left(J\left(x_{0}, y_{0}\right)\right)=\left|\begin{array}{ll}
\frac{\partial f_{1}}{\partial x} & \frac{\partial f_{1}}{\partial y} \\
\frac{\partial f_{2}}{\partial x} & \frac{\partial f_{2}}{\partial y}
\end{array}\right|_{x=x_{0}, y=y_{0}} \neq 0
$$

$\Delta x, \Delta y$ can be calculated by the following equations

$$
\left\{\begin{array}{l}
\Delta x \frac{\partial f_{1}\left(x_{0}, y_{0}\right)}{\partial x}+\Delta y \frac{\partial f_{1}\left(x_{0}, y_{0}\right)}{\partial y}=-f_{1}\left(x_{0}, y_{0}\right) \\
\Delta x \frac{\partial f_{2}\left(x_{0}, y_{0}\right)}{\partial x}+\Delta y \frac{\partial f_{2}\left(x_{0}, y_{0}\right)}{\partial y}=-f_{2}\left(x_{0}, y_{0}\right)
\end{array}\right.
$$

Then after updating $x_{0}=x_{0}+\Delta x, y_{0}=y_{0}+\Delta y$, we repeat those operations as above until $\max (|\Delta x|,|\Delta y|)<\varepsilon$, where $\varepsilon$ is the given precision.

The position error can be expressed as follows

$$
\left\{\begin{array}{l}
\Delta x_{p}=x_{p}{ }^{\prime}-x_{p} \\
\Delta y_{p}=y_{p}{ }^{\prime}-y_{p}
\end{array}\right.
$$

where $\left(x_{p}{ }^{\prime}, y_{p}{ }^{\prime}, 0\right)$ is the position that be obtained using Newton iteration method. The location error can be defined as

$$
\Delta E=\sqrt{\Delta x_{\mathrm{p}}^{2}+\Delta y_{p}^{2}}
$$

\section{SIMULATION RESULTS}

\begin{tabular}{|c|c|c|}
\hline Parameters & Transmitter values & Receiver values \\
\hline Velocity in $\mathrm{X}$ direction & $140.9 \mathrm{~m} / \mathrm{sec}$ & $76.6 \mathrm{~m} / \mathrm{sec}$ \\
\hline Velocity in $\mathrm{Y}$ direction & $51.3 \mathrm{~m} / \mathrm{sec}$ & $65.0 \mathrm{~m} / \mathrm{sec}$ \\
\hline Velocity in $\mathrm{Z}$ direction & \multicolumn{2}{|c|}{$0 \mathrm{~m} / \mathrm{sec}$} \\
\hline Altitude & $8000 \mathrm{~m}$ & $500 \mathrm{~m}$ \\
\hline Center frequency & \multicolumn{2}{|c|}{$\mathrm{X}$ Band } \\
\hline Range bandwidth & \multicolumn{2}{|c|}{$80 \mathrm{MHZ}$} \\
\hline Doppler bandwidth & \multicolumn{2}{|c|}{$2500 \mathrm{HZ}$} \\
\hline Slope ranges & $50000 \mathrm{~m}$ & $9000 \mathrm{~m}$ \\
\hline Squint angles & $35.5^{\circ}$ & $40.0^{\circ}$ \\
\hline velocity & $150 \mathrm{~m}$ & $100 \mathrm{~m}$ \\
\hline
\end{tabular}

In this section, we present some simulation results to verify the location algorithms proposed in this paper. Fig. 3 shows how the simulated scenario is designed. It is preset with six strong ground targets, i.e. T1 T6, and thousands of point targets with different radar cross-section values to form a background map. Table 1 shows the simulation parameters.

TABLE 1. SiMULATION PARAMETERS 

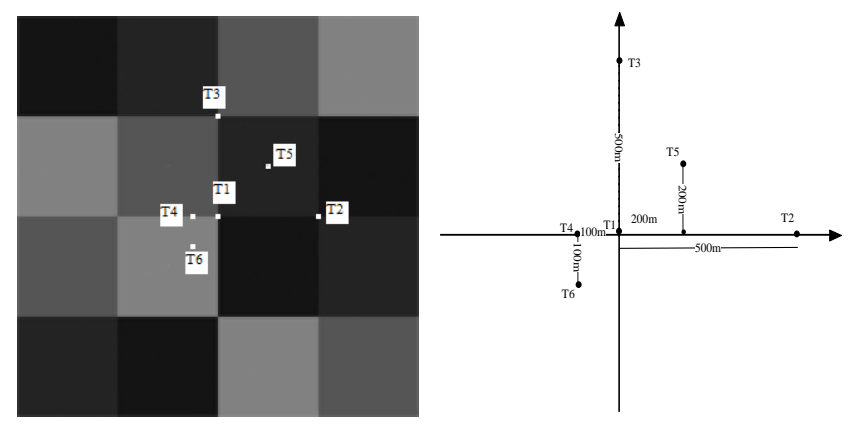

Fig. 3. Placement and positions of the point-targets in the simulated scenario

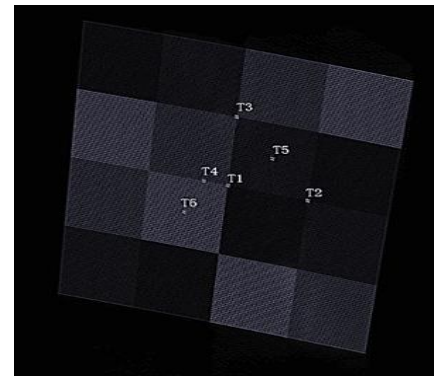

(a)

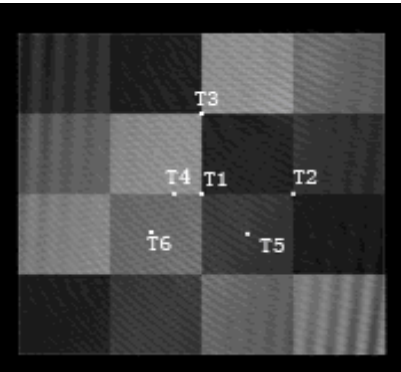

(b)
Fig. 4. Imaging results (a) using the LRD processing and (b) after geometric distortion correction

The imaging results are shown in Fig. 4. As depicted in Fig. 4(a), the difference between the range-resolution and the azimuth-resolution causes geometric distortion, which affects the targets location accuracy. The geometric distortion and the location of the targets can be unified. If each pixel point can be confirmed, then the geometric distortion correction also can be accomplished [12]. The BiSAR image after geometric distortion correction is shown in Fig. 4(b).

After imaging, we locate the point targets using the image in the simulation based on the Newton iteration method. According to the steps of the Newton iteration method, the location results and the geolocation errors $\Delta E$ can be obtained as shown in Table 2.

TABLE 2. ANALYSIS OF LOCATION RESULTS

\begin{tabular}{ccccc}
\hline \hline Targets & $\begin{array}{c}\text { Actual } \\
\text { Position(m) }\end{array}$ & $\begin{array}{c}\text { Coordinates in } \\
\text { Image (pixel) }\end{array}$ & $\begin{array}{c}\text { Calculated } \\
\text { Position }(\mathrm{m})\end{array}$ & $\Delta \mathrm{E}(\mathrm{m})$ \\
\hline T1 & $(0,0)$ & $(2048,1024)$ & $(0.0,0.0)$ & 0.0 \\
T2 & $(0,500)$ & $(2092,742)$ & $(0.22,499.3)$ & 0.839 \\
T3 & $(500,0)$ & $(2290,1146)$ & $(500.5,-1.24)$ & 1.338 \\
T4 & $(0,-100)$ & $(2039,1081)$ & $(-0.42,-100)$. & 0.960 \\
T5 & $(200,200)$ & $(2186,959)$ & $(199.9,198.7)$ & 1.2797 \\
T6 & $(-100,-100)$ & $(1981,1058)$ & $(-100.03,-100.6)$ & 0.693 \\
\hline \hline
\end{tabular}

\section{CONCLUSION}

It is well known in optimization theory that Newton method has the character of robustness and higher rate of convergence. The only real drawback of using Newton is the algebraic complexity and computational cost of assembling the derivative terms of the Jacobin matrix. From the analysis and given simulation results, we find that the LRD algorithm is effective for the Bistatic SAR imaging at the general case of large squint angles, nonparallel trajectories and different velocities. However, the LRD algorithm does not work well when the imaging scene becomes very large. Future work could be related to reduce the effect using the digital spotlight techniques.

\section{ACKNOWLEDGMENT}

This work was supported by the Aeronautical Science Foundation of China(No.20112052025).

\section{REFERENCES}

[1] Otmar Loffeld,Holger Nies,Valerij Peters,and Stefan Knedlik, "Models and Useful Relations for Bistatic SAR Processing", IEEE TRANS.ON GEOSCIENCE AND REMOTE SENSING,VOL.42,No.10,Oct. 2004.

[2] Li YanPing,Zhang ZhenHua, "A Novel Range Migration Algorithm for General Bistatic SAR Imgaing Based on Series Reversion and Numerical Computation", Vol.30, No.12, Journal and Electronics and Information Technology, 2008.

[3] Koba Natroshvili,Otmar Loffeld,Amaya Medrano Ortiz and Stefan Knedlik, "Focusing of General Bistatic SAR Configuration Data With 2-D Inverse Scaled FFT", IEEE TRANSACTIONS ON GEOSCIENCE AND REMOTE SENSING,VOL.44,NO.10,Oct 2006.

[4] Li Yong, Zhu Dai-yin, Zhu Zhao-da "Study on High Resolution Overlapped Sub aperture Imaging Algorithm for High Squint Airborne SAR Processing", Journal of Electronics and Information technology, Vol.28, No.9, Sept. 2006.

[5] Claudio Paniconi Mario Putti, “A comparison of Picard and Newton iteration in the numerical solution of multidimensional variably saturated flow problems" . WATER RESOURCES RESEARCH, VOL. 30, NO. 12, PP. 3357-3374, 1994

[6] R.Peris,A.Marquina,V.Candela, "The convergence of the perturbed Newton method and its application for ill-conditioned problems", Applied Mathematics and Computation, 218 pp. 2988-3001, 2011

[7] Cao Xiangwu,Huang Guangming, "Study on a Fast Target Location Method for Airborne SAR and Location Precision Analysis", Modern Radar,VOL.26, No.9, Sept. 2004.

[8] Geng X P,Yan H H,Wang Y F. "A two-dimensional spectrum model for general bistatic SAR". IEEE Trans. on Geoscience and Remote Sensing, 2008, pp.2216-2223.

[9] Qiu X L,Hu D H,Ding C B."An improved NLCS algorithm with capability analysis for one-stationary BISAR", IEEE Trans. on Geoscience and Remote Sensing, 2008, pp.3179-3186.

[10]Yew Lam Neo,Frank H.Wong, and Ian G.Cumming, "Processing of Azimuth-Invariant Bistatic SAR Data Using the Range-Doppler Algorithm", IEEE Trans. on Geoscience and Remote Sensing, Vol.46, No.1, January 2008.

[11]D.D'Aria,A.Monti Guarnieri,and F.Rocca, "Focusing bistatic synthetic aperture radar using dip move out," IEEE Trans. on Geoscience and Remote Sensing, vol,42, No.7, pp.1362-1376, Jul. 2004.

[12]Blacknell, D.; Freeman, A.; White, R.G.; Wood, J.W., "The Prediction of Geometric Distortions in Airborne Synthetic Aperture Radar Imagery from Autofocus Measurements", IEEE Trans. on Geoscience and Remote Sensing,Vol.GE-25, No.6, November 1987. 